\title{
Expression profiles of MMP-1 and TIMP-1 in lumbar intervertebral disc degeneration
}

\author{
B. Deng ${ }^{1 *}$, J.Z. Ren ${ }^{1 *}$, X.Q. Meng ${ }^{1}$, C.G. Pang1', G.Q. Duan ${ }^{2}$, J.X. Zhang ${ }^{3}$, \\ H. Zou' ${ }^{1}$, H.Z. Yang ${ }^{4}$ and J.J. Ji ${ }^{1}$
}

1'Department of Bone Surgery, Zoucheng People's Hospital, Zoucheng, Shandong Province, China

${ }^{2}$ Department of Bone Surgery, Affliated Hospital of Jining Medical College, Jining, Shandong Province, China

${ }^{3}$ Department of Spine Surgery,

Shandong Province Hospital of Traditional Chinese Medicine, Jinan,

Shandong Province, China

${ }^{4}$ Shandong University School of Medicine, Jinan, Shandong Province, China

*These authors contributed equally to this study.

Corresponding author: J.J. Ji

E-mail: haadshuiyahoo@126.com

Genet. Mol. Res. 14 (4): 19080-19086 (2015)

Received August 18, 2015

Accepted October 24, 2015

Published December 29, 2015

DOI http://dx.doi.org/10.4238/2015.December.29.16

ABSTRACT. Lumbar intervertebral disc degeneration (IDD) is a common clinical pathology and has become a focus for research in recent years. Matrix metalloproteinases (MMPs) are enzymes responsible for the degradation of almost all extracellular matrix proteins (ECM). The over-expression of MMPs or tissue inhibitors of metalloproteinases (TIMPs) may disrupt the dynamic balance of the ECM. Therefore, in the current study, the expression levels of MMP-1 and TIMP-1 in lumbar IDD patients were evaluated in an attempt to elucidate their role in IDD pathogenesis and progression. In total, 60 IDD patients were recruited as the experimental group, along with 20 cases of lumbar vertebral injury without disc degeneration as the control group. Preoperative venous blood samples were collected, and intervertebral disc tissues were collected from the lesion during surgery. Serum and tissue levels 
of MMP-1 and TIMP-1 were quantified by enzyme-linked immunosorbent assay and immunohistochemical staining, respectively. Serum and tissue MMP-1 levels in IDD patients were significantly higher than those in the control group $(P<0.05)$. Additionally, sub-group analysis revealed that severe IDD patients had higher MMP-1 levels compared with mild or moderate IDD patients $(P<0.05)$. However, there were no significant differences in TIMP1 levels in either the serum or tissues of IDD patients compared to patients in the control group $(P>0.05)$. These results demonstrate that MMP-1 expression is increased in IDD, with higher expression observed in more severe cases, whereas TIMP-1 expression was similarly expressed in both normal and degenerated discs.

Key words: Lumbar intervertebral disc degeneration; Matrix metalloproteinase-1; Tissue inhibitor of metalloproteinase-1

\section{INTRODUCTION}

Lumbar intervertebral disc herniation is the most common form of intervertebral disc degeneration (IDD), and is manifested by bulging of the central nucleus pulposus granulation of the fibrous ring, and tissue fibrosis (Li et al., 2010; Samartzis et al., 2012). Matrix metalloproteinases (MMPs) participate in both synthesis and degradation of the extracellular matrix (ECM), thereby regulating intervertebral disc dynamics (Ozkanli et al., 2015). Tissue inhibitors of metalloproteinases (TIMPs) are specific inhibitors of MMPs, and are known to be involved in IDD (Leung et al., 2011). Therefore, it is possible that the balance between MMPs and TIMPs is involved in the pathogenesis and progression of IDD. This study investigated the expression profile of MMP-1 and TIMP-1 in lumbar IDD patients as an attempt to elucidate the function of these two ECM regulating factors in IDD pathology.

\section{MATERIAL AND METHODS}

\section{Patient information}

In total, 60 lumber IDD patients (30 males and 30 females between 22 and 55 years old with an average age of $35.6 \pm 1.8$ years) were recruited for this study as the experimental group. All patients were diagnosed with IDD via magnetic resonance imaging (MRI) as categorized by the following three stages: stage I, mildly decreased intervertebral disc signals; stage II, moderately decreased intervertebral disc signals; and stage III, severely decreased intervertebral disc signals. Another 20 patients (10 males and 10 females, aged between 21 and 56 years old with an average of $34.7 \pm 2.2$ years) each with a lumbar spine injury but normal intervertebral disc height and signal as determined by MRI. There were no statistically significant differences regarding gender or age between the experimental and control groups. The experimental protocol was pre-approved by the ethical committee of our hospital and written informed consent was obtained from all patients and healthy volunteers.

\section{Serum protein level assays}

Venous blood samples drawn from patients were centrifuged to separate and isolate the 
serum, which was then frozen until further use. Before the enzyme-linked immunosorbent assay (ELISA), all samples and reagents were pre-warmed to room temperature. ELISA kits for MMP-1 and TIMP-1 were purchased from Roche (Switzerland) and ELISAs were performed according to manufacturer instructions. Five replicates were added to the ELISA plates for each sample dilution, followed by washing, development, and termination. Optical density (OD) values were measured at $450 \mathrm{~nm}$. The concentrations of the target proteins were determined by a reference curve that was plotted using kit-provided standards.

\section{Immunohistochemical (IHC) staining}

Nucleus pulposus tissue samples were collected from patients of two groups, and were cut into $0.5 \mathrm{~cm} \times 0.5 \mathrm{~cm} \times 0.5 \mathrm{~cm}$ cubes. After fixation in $10 \%$ formalin, dehydration, embedding in wax, de-waxing, and rehydration, antigen retrieval was performed using citric acid buffer. The slices were then processed using $3 \%$ hydrogen peroxidase to quench endogenous peroxidase activity. Non-specific binding sites were blocked by the addition of goat serum. Slices were incubated with a primary antibody and secondary antibodies conjugated with horseradish peroxidase for 1 hour and $10 \mathrm{~min}$, respectively. Slices were then dehydrated and mounted with coverslips. Images were captured by an inverted light-field microscope (Olympus, Japan).

\section{Analysis of IHC staining}

Negative controls were incubated with PBS buffer instead of the primary antibody in parallel. After image capture, 5 image fields were randomly selected from one slide. Positive signals for MMP-1 or TIMP-1 were observed as dark brown/yellow brown coloring in the cytoplasm or at the cell membrane but not in the nucleus. The percentage of positive cells in one field was calculated and averaged among the five fields. The grade of staining was defined as: 1$)$ negative $(-),<10 \%$ cells stained positive; 2 ) weak positive $(+), 11-25 \%$ cells stained positive; 3 ) positive $(++), 26-50 \%$ cells stained positive; and 4) strong positive $(+++),>50 \%$ cells with positive signals. The overall positive rate was defined as the number of cases with grade (2), (3) or (4) / total number of cases in one group.

\section{Statistical analysis}

The SPSS 17.0 software was used for statistical analyses of all collected data, of which numeration data were compared using chi-square tests while measurement data were analyzed via analyses of variance (ANOVA). Between-group comparisons were performed using an LSD test. All measurement data are reported as means \pm standard deviation (SD). Statistical significance was defined as $\mathrm{P}<0.05$.

\section{RESULTS}

\section{Serum levels of MMP-1 and TIMP-1}

ELISA was used to quantify serum MMP-1 and TIMP-1 levels in IDD patients and control individuals. The results (Figure 1A) showed significantly elevated MMP-1 levels in the IDD patient group $(P=0.015, P=0.011, P=0.001)$. A further comparison among three sub-groups of IDD revealed that patients with stage III (severe) IDD had significantly higher MMP-1 serum levels 
compared to patients with stage I (mild) or stage II (moderate) $(P=0.014, P=0.008)$. However, there were no significant differences in the serum TIMP-1 levels between any of the three IDD stages or control group ( $P>0.05$, Figure 1B).

A

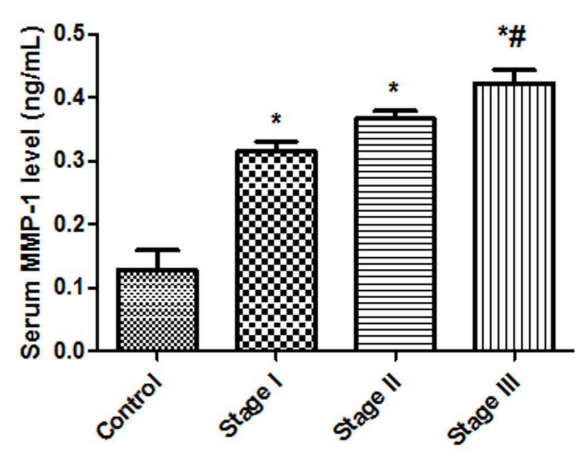

B

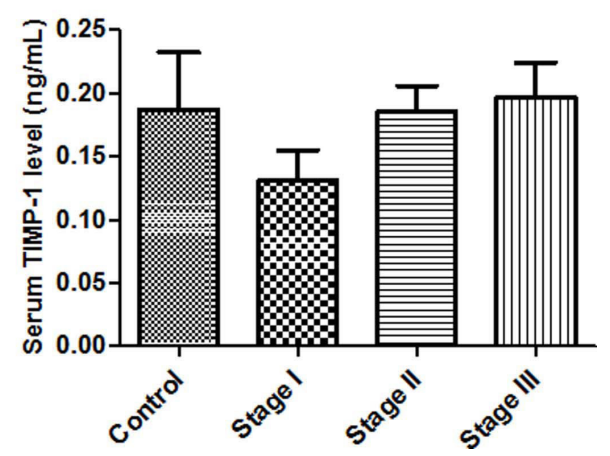

Figure 1. Serum levels of MMP-1 (A) and TIMP-1 (B). Serum levels were compared among control and all three stages of IDD. MMP-1 concentration was elevated in IDD patients, with the highest level observed in stage III (sever) IDD patients. No significant differences were observed in TIMP-1 expression. Significance was determined by one-way analysis of variance (ANOVA) followed by the post hoc LSD test. ${ }^{*} \mathrm{P}<0.05$ compared to controls; $\mathrm{P}<0.05$ compared to stage I and stage II IDD patients.

\section{MMP-1 and TIMP-1 protein expression in tissues}

We further used IHC analyses to examine the expression profiles of MMP-1 and TIMP1 proteins in the patient and control groups. As revealed by the IHC images, control tissues had irregular cell shapes, with blue round nuclei, pink cytoplasm, and evenly distributed ECM. Conversely, IDD patients had round-shaped nuclei, cytoplasmic vacuoles, increased ECM with disarranged fibers, and significantly elevated cell proliferation (Figure 2). Both MMP-1 and TIMP-1 were expressed in the membrane and cytoplasm and were observed as brown and yellow granules. Further analysis revealed that the percent positive rates of MMP-1 in all three IDD stages were significantly higher than that in the control group (Table 1, P < 0.05). Furthermore, stage III patients had the highest $(95 \%)$ positive rates of MMP-1, which was significantly higher than those of stage I or stage II patients $(\mathrm{P}<0.05)$. TIMP-1 positive cells were identified in the patient and control groups but no significant differences in the positive rates were observed $(P>0.05)$.

\section{DISCUSSION}

As a prominent pathological change associated with lumbar intervertebral disc herniation, IDD is clinically manifested by symptoms including low back pain and fatigue. The major component of the ECM in intervertebral discs is proteoglycan, which can interact with collagen to form a stable matrix to lubricate and decrease pressures in the intervertebral space (Wu et al., 2010; Mavrogonatou et al., 2014). The major types of collagen in the intervertebral discs are type I and type II. For example, in the fibrous ring, collagen fibers mainly consist of type I and type II, in 


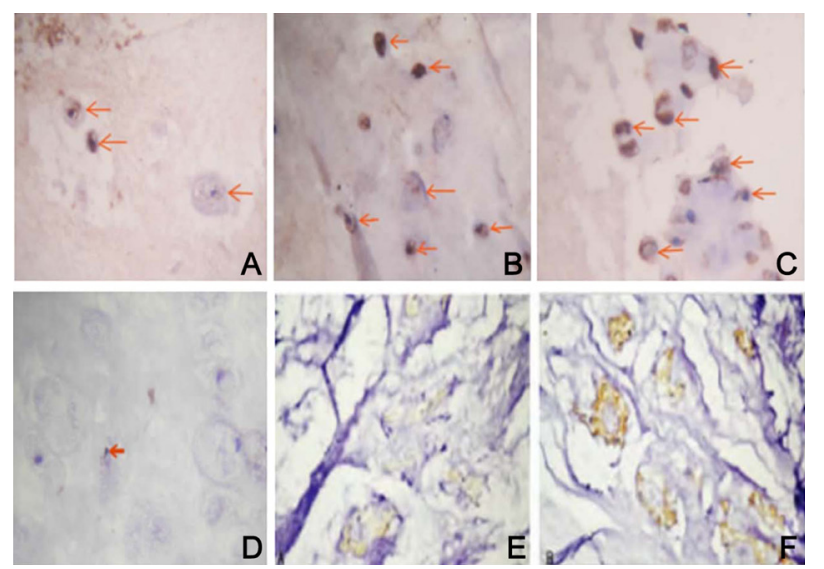

Figure 2. MMP-1 expression in intervertebral disc tissues. (A) and (D), weak positive (+); (B) and (E), positive (++); (C) and $(\mathbf{F})$, strong positive $(+++)$. Images are shown at 400X magnification.

Table 1. MMP-1 and TIMP-1 expression in patient and control tissues.

\begin{tabular}{|c|c|c|c|c|c|c|c|c|c|}
\hline \multirow[t]{2}{*}{ Group/Stage } & \multirow[t]{2}{*}{$\mathrm{N}$} & \multicolumn{4}{|c|}{ MMP-1 } & \multicolumn{4}{|c|}{ TIMP-1 } \\
\hline & & - & $+/++$ & +++ & Positive rate (\%) & - & $+/++$ & +++ & Positive rate $(\%)$ \\
\hline IDD patients & 60 & & & & & & & & \\
\hline Stage I & 20 & 10 & 7 & 3 & $50^{*}$ & 14 & 5 & 1 & 30 \\
\hline Stage II & 20 & 6 & 9 & 5 & $70^{*}$ & 13 & 6 & 1 & 35 \\
\hline Stage III & 20 & 1 & 11 & 8 & $95^{\star \#}$ & 12 & 6 & 2 & 40 \\
\hline Control & 20 & 18 & 2 & 0 & 10 & 10 & 10 & 0 & 20 \\
\hline
\end{tabular}

${ }^{*} \mathrm{P}<0.05$ compared to control; ${ }^{\# P}<0.05$ compared to stage I and stage II IDD patients.

addition to minor amounts of type III, V, VI, and IX. In the nucleus pulposus, collagens are mainly type II but also include type VI, IX, and XI (Yuan et al., 2011; Canbay et al., 2013).

Recent studies have revealed the important roles of cytokines and inflammatory factors in the pathogenesis of IDD (Gomis-Rüth, 2009). The synthesis and degradation of the ECM in intervertebral disc tissues are known to be regulated by MMPs and are closely related to IDD (Zawilla et al., 2014). The up-regulation of MMPs may lead to elevated ECM degradation, alternative collagen components, breakdown of the fibrous ring, and intervertebral tissue damage, finally leading to the herniation of the nucleus pulposus (Rastogi et al., 2013). Previous reports have attributed the major reason for over-degradation of the intervertebral disc matrix as the imbalance between MMP and TIMP (Carvalho et al., 2009; Kimura et al., 2010). MMP-1, as one kind of collagenase, is able to degrade collagens including type I, II, III, VII, and VIII (Pockert et al., 2009). As the inhibitors of MMPs, TIMPs can suppress the activity of MMPs by the formation of one-to-one complexes (Gruber et al., 2012; Zou et al., 2014).

In the current study, we categorized all recruited IDD patients into three stages of IDD (mild, moderate, and severe) according to MRI signal strength. Preoperative venous blood samples were collected for quantification of serum MMP-1 and TIMP-1 levels. Results showed that IDD patients had significantly higher serum MMP-1 levels compared to those in the control group. Further between-group comparisons revealed that severe IDD patients had higher MMP-1 levels compared to mild or moderate IDD patients. The levels of TIMP, however, were not significantly 
different between patients and control individuals. IHC analysis revealed similar results: 1) severe IDD patients had significantly higher MMP-1 positive rates compared to moderate or mild IDD patients; 2) All IDD patients had a greater number of MMP-1 positive cells compared to controls; and 3) TIMP levels were similar across all groups. Taken together, these results suggest that MMP1 expression is elevated in IDD patients especially in patients with more severe disc degeneration, while TIMP is expressed similarly in both normal and IDD tissues. Our results are supported by other studies that have shown that herniated intervertebral disc tissues had high levels of MMPs, and that expression levels were positively correlated with IDD (Vo et al., 2013). Study with both human and animal model systems has demonstrated that surgical injury to the intervertebral disc leads to elevated MMP-1 expression (Baillet et al., 2013), suggesting the participation of MMP-1 in ECM degradation, which can be suppressed by TIMP to slow the occurrence of IDD. Other studies have attributed IDD to the decrease of cytokines and elevated inflammatory factor release, both of which lead to higher MMP-1 and lower TIMP-1 (Yurube et al., 2012). Although both degenerative and normal intervertebral disc tissues express MMP-1 and TIMP-1, in degenerative intervertebral disc tissues, significantly elevated MMP-1 and decreased TIMP-1 cause an imbalance that leads to ECM degradation. The decreased TIMP-1 levels cannot inhibit the degradation of the ECM by MMP-1, which leads to inelasticity of the intervertebral disc and herniation (Furtwangler et al., 2013). All of these prior studies are consistent with the results herein.

In summary, MMP-1 is prominently expressed during lumbar IDD, implying a positive correlation with IDD severity. TIMP-1, however, is expressed similarly in both normal and degenerative intervertebral disc tissues. The imbalance of MMP-1 and TIMP-1, and resultant degradation of the intervertebral disc ECM, may underlie the pathogenesis of IDD. Therefore, the regulation of the relative expression of MMPs and TIMPs, and subsequent ECM metabolism regulation, may provide new strategies in treating intervertebral disc herniation, although further mechanistic investigations are needed.

\section{Conflicts of interest}

The authors declare no conflict of interest.

\section{ACKNOWLEDGMENTS}

We thank the anonymous reviewers for reviewing this manuscript.

\section{REFERENCES}

Baillet A, Grange L, Trocme C, Caudroy S, et al. (2013). Differences in MMPs and TIMP-1 expression between intervertebral disc and disc herniation. Joint Bone Spine 80: 341-2.

Canbay S, Turhan N, Bozkurt M, Arda K, et al. (2013). Correlation of matrix metalloproteinase-3 expression with patient age, magnetic resonance imaging and histopathological grade in lumbar disc degeneration. Turk Neurosurg. 23: 427-33.

Carvalho RV, Ogliari FA, De Souza AP, Silva AF, et al. (2009). 2-Hydroxyethyl methacrylate as an inhibitor of matrix metalloproteinase-2. Eur. J. Oral Sci. 117: 64-67.

Furtwangler T, Chan SC, Bahrenberg G, Richards PJ, et al. (2013). Assessment of the matrix degenerative effects of MMP3, ADAMTS-4, and HTRA1, injected into a bovine intervertebral disc organ culture model. Spine (Phila Pa 1976) 38: E1377-87.

Gomis-Rüth FX (2009). Catalytic domain architecture of metzincin metalloproteases. J. Biol. Chem. 284: $15353-15357$.

Gruber HE, Hoelscher GL, Ingram JA, Hanley EN Jr., et al. (2012). Matrix metalloproteinase-26, a novel MMP, is constitutively 
expressed in the human intervertebral disc in vivo and in vitro. Exp. Mol. Pathol. 92: 59-63.

Kimura K, Cheng XW, Nakamura K, Inoue A, et al. (2010). Matrix metalloproteinase-2 regulates the expression of tissue inhibitor of matrix metalloproteinase-2. Clin. Exp. Pharmacol. Physiol. 37: 1096-1101.

Leung VY, Tam V, Chan D, Chan BP, et al. (2011). Tissue engineering for intervertebral disk degeneration. Orthop. Clin. North. Am. 42: 575-83.

Li FC, Zhang N, Chen WS, Chen QX, et al. (2010). Endplate degeneration may be the origination of the vacuum phenomenon in intervertebral discs. Med. Hypotheses. 75: 169-71.

Mavrogonatou E, Angelopoulou MT \& Kletsas D (2014). The catabolic effect of TNFalpha on bovine nucleus pulposus intervertebral disc cells and the restraining role of glucosamine sulfate in the TNFalpha-mediated up-regulation of MMP3. J. Orthop. Res. 32: 1701-7.

Ozkanli S, Kaner T, Efendioglu M, Basaran R, et al. (2015). The relation of matrix metalloproteinase 1, 2, 3 expressions with clinical and radiological findings in primary and recurrent lumbar disc herniations. Turk. Neurosurg. 25: 111-6.

Pockert AJ, Richardson SM, Le Maitre CL, Lyon M, et al. (2009). Modified expression of the ADAMTS enzymes and tissue inhibitor of metalloproteinases 3 during human intervertebral disc degeneration. Arthritis \& Rheum. 60: 482-491.

Rastogi A, Kim H, Twomey JD and Adam H Hsieh (2013). MMP-2 mediates local degradation and remodeling of collagen by annulus fibrosus cells of the intervertebral disc. Arthritis Res. Ther. 15: R57.

Samartzis D, Karppinen J, Chan D, Luk KD, et al. (2012). The association of lumbar intervertebral disc degeneration on magnetic resonance imaging with body mass index in overweight and obese adults: a population-based study. Arthritis Rheum 64: 1488-96.

Vo NV, Hartman RA, Yurube T, Jacobs LJ, et al. (2013). Expression and regulation of metalloproteinases and their inhibitors in intervertebral disc aging and degeneration. Spine J. 13: 331-41.

Wu WP, Jiang JM, Qu DB, Wei QZ, et al. (2010). [Expression of hypoxia-inducible factor-1alpha and matrix metalloproteinase-2 in degenerative lumbar intervertebral disc]. Nan Fang Yi Ke Da Xue Xue Bao 30: 1152-5.

Yuan Y, Zhao J, Li Y-M, Wang X, et al. (2011). Expression and significance of NF-KB, MMP-1/TIMP-1 in intervertebral disc degeneration. Shandong Med. J. 19: 004.

Yurube T, Takada T, Suzuki T, Kakutani K, et al. (2012). Rat tail static compression model mimics extracellular matrix metabolic imbalances of matrix metalloproteinases, aggrecanases, and tissue inhibitors of metalloproteinases in intervertebral disc degeneration. Arthritis Res. Ther. 14: R51.

Zawilla NH, Darweesh H, Mansour N, Helal S, et al. (2014). Matrix metalloproteinase-3, vitamin D receptor gene polymorphisms, and occupational risk factors in lumbar disc degeneration. J. Occup. Rehabil. 24: 370-81.

Zou J, Huang GF, Zhang Q, Gao Y, et al. (2014). Effects of electroacupuncture stimulation of “Jiaji” (EX-B 2) on expression of matrix metalloproteinase-13 and tissue inhibitor of metalloproteinase-1 in intervertebral disc tissue in rabbits with lumbar intervertebral disc degeneration. Zhen Ci Yan Jiu 39: 192-7. 